\title{
Second-order conditioning during sleep
}

\author{
ELIZABETH HENNEVIN and BERNARD HARS \\ Laboratoire de Neurobiologie de l'Apprentissage et de la Mémoire \\ C.N.R.S., URA 1491, Université Paris-Sud, Orsay, France
}

\begin{abstract}
To test whether or not the pairing of two external stimuli during sleep could induce a change in a behavioral response during the waking state, we used a second-order conditioning procedure and subsequently tested its effectiveness in a lick suppression response. After lick training, waking rats underwent first-order conditioning with low-level electrotactile stimulation to the ear (ETS) signaling a footshock. On the following day, the animals were exposed while awake or asleep to second-order pairing with a tone signaling ETS delivery. They were subsequently tested for lick suppression to the tone. The results showed that animals that had received tone-ETS pairings during either paradoxical sleep or slow-wave sleep exhibited lick suppression to the tone. This response was less pronounced than that of subjects who had received pairing during waking. But it was strong enough to be maintained over all three test sessions, which attests to its reliability. The nature of the process that took place during sleep as a result of second-order pairing is discussed.
\end{abstract}

Most of the studies intended to determine the cerebral conditions that control encoding, storage, and retrieval processes refer to the waking state. Yet the study of how information processing is affected by natural changes in cerebral activity that occur during sleep may provide insights about the neurophysiological configurations that are required for information processing to take place. In other words, sleep can be used as a tool for understanding the mechanisms of learning and memory. Moreover, the study of information-processing ability during the two states of sleep-slow-wave sleep (SWS) and paradoxical sleep (PS)-may contribute toward our understanding of their functional meaning, which still remains elusive.

The ability of a sleeping organism to process information remains an open question. In research on animals, studies have addressed the possibility that already-significant stimuli can be detected during sleep. Indeed, evidence has been presented that discriminative responses can be observed during SWS, depending on stimulus relevance (Buendia, Sierra, Goode, \& Segundo, 1963; Ciancia, Trigona-Leisinger, \& Bloch, 1980; Halperin \& Iorio, 1981; Lelord \& Maho, 1969; Rowland, 1957; Sano, Matsumoto, \& Ishikawa, 1982; Sasaki \& Yoshii, 1984; Segundo, Roig, \& Sommer-Smith, 1959; Yehuda, Chorover, \& Carasso, 1979). On the other hand, despite limited responsiveness to external stimuli during PS (Dillon \& Webb, 1965; Trigona, Ciancia, \& Bloch, 1968; Van Twyver \& Garrett, 1972), several lines of evidence suggest that information coming from the external world can likewise be processed during this state of sleep. First, there are some reports of lowered arousal thresholds following conditioning relative to when the stimulus is neu-

Correspondence should be addressed to Elizabeth Hennevin, NAM, C.N.R.S., Université Paris-Sud, Bat. 446, 91405 Orsay Cedex, France. tral (Siegel \& Langley, 1965). Further, we have shown that when a cue stimulus that has acquired significance through conditioning in wakefulness is given as a reminder in PS, subsequent retention performance is modified. Presentation of the same stimulus has no effect when the stimulus has not previously been associated with the learning task (Hars \& Hennevin, 1990; Hars, Hennevin, \& Pasques, 1985). To account for the efficacy of cuing during PS, we must assume that the cue stimulus is detected during PS. This has been substantiated by our recent demonstration that a hippocampal conditioned cellular response acquired during waking can be evoked by presentation of the conditioned stimulus in PS (Maho, Hennevin, Hars, \& Poincheval, 1991). Thus, all of these studies indicate that a behaviorally significant stimulus can be recognized by a sleeping organism. This suggests that, even during lowered levels of vigilance, some sort of scanning mechanism operates, gating input so that only important information reaches the organism.

On the other hand, very little is known about the possibility that acquisition processes may take place during sleep. One kind of learning that seems to occur during sleep is habituation (Johnen, 1987; Rudolph, 1987; Yehuda et al., 1979). There are also some reports that extinction can develop during sleep (Izquierdo, Wyrwicka, Sierra, \& Segundo, 1965; Oniani \& Lortkipanidze, 1985). Few studies, however, have been designed to assess the possibility that initially nonsignificant stimuli can acquire significance during sleep. In some cases, positive results have been obtained in SWS (Delacour, 1980, 1982; Izquierdo et al., 1965), but they are inconclusive. The main problem in such studies is that the experimental paradigm has generally involved conditioned arousal as the conditioned response; consequently, it is difficult to determine in which vigilance state information has been processed. The most convincing evidence for new association formed during sleep has been provided by Maho and Bloch (in 
press), who have developed a conditioning paradigm in which the conditioned stimulus (CS) and the unconditioned stimulus (UCS) are nonawakening intracerebral stimulations. An increase in hippocampal multiunit activity to CS presentation is taken as the conditioned response. Maho and Bloch show that pairing the CS with the UCS during PS allowed the conditioned cellular response to develop, almost as easily as during waking. No conditioning was established during SWS.

The present study was an attempt to expand these findings. Our main purpose was to determine whether pairing two external sensory stimuli during sleep could induce a change in subsequent behavior of the waking animal.

We used a second-order conditioning procedure to address this question. As Pavlov (1927) first demonstrated, once a stimulus is associated with a reinforcer (the UCS), that stimulus takes on the ability to establish a conditioned response to other initially neutral stimuli by which it is itself signaled. It has been shown not only that secondorder learning is robust and reliable, but also that secondorder techniques can reveal conditioned responses in experimental protocols that have typically resulted in poor or no conditioning (Rescorla, 1980). In a second-order conditioning procedure, a neutral stimulus (S2) is regularly followed by a stimulus (S1) that has reinforcing power only because it has previously been paired with a UCS. The advantages of this procedure are clear when one wants to pair stimuli during sleep. First, unlike in simple Pavlovian conditioning, one does not deliver a biologically significant UCS, the occurrence of which would be likely to awaken the animal. Second, unlike in a sensory preconditioning paradigm, it provides the possibility to present an already-significant stimulus (S1), which increases the probability of detection by the sleeping organism. To subsequently assess the effect of second-order pairing in waking animals, we used a conditioned emotional response paradigm (see Estes \& Skinner, 1941). The effect of second-order pairing was measured by the extent to which presentation of the second-order stimulus brought about suppression of drinking behavior.

The first-order stimulus consisted of low-level electrotactile stimulations (ETSs) delivered to the ear. In previous experiments, it has been shown that such stimulation can serve as CS in active avoidance conditioning (Hars \& Hennevin, 1987; Hars et al., 1985). In a foodreinforced maze-learning task, during which ETS was used as an intratask cue, it has been demonstrated that delivery of ETS in the maze has no effect on learning performance (Hars \& Hennevin, 1990). These results show that ETS by itself has no reinforcing power. The secondorder stimulus was a tone. The basic experimental procedure included four phases. Phase 1 constituted a preliminary training phase designed to establish a baseline rate of licking. Phase 2 was a first-order conditioned suppression training, during which ETS was paired with foot- shock. During Phase 3, a second-order conditioning procedure was conducted by pairing a tone with ETS. Phase 4 was the testing phase, in which tone presentation was used to measure the effectiveness of the preceding procedure on suppression of licking. Phases 1,2 , and 4 were always conducted while the subjects were awake. Phase 3 , the phase of interest, was conducted either with waking or with sleeping subjects.

\section{EXPERIMENT 1}

Contemporary discussions of classical conditioning have increasingly stressed the importance of the context in which learning and performance occur. It is clear that learned behavior is modulated by the context in which it is learned and tested. Contextual modulations of learning and performance have been observed in both appetitive and aversive paradigms, in which both interoceptive and exteroceptive stimuli have been used (for reviews, see Balsam, 1985; Overton, 1985; Rescorla, Durlach, \& Grau, 1985). All basic learning phenomena that have been studied have been shown to be affected by contextual manipulations; for instance, the effects of conditioning, extinction, habituation, and latent inhibition may all be at least partly confined to the context in which animals have received these experimental treatments (see, e.g., Bouton \& Bolles, 1985; Hall \& Channell, 1985; Lovibond, Preston, \& Mackintosh, 1984).

This raises the question of the effects that might result from contextual changes inherent in a paradigm with second-order pairing given during sleep, after which its efficacy will be tested during waking. Obviously, such a procedure implies changes in the internal context between the pairing and testing phases. Furthermore, it also requires changes of external context. Indeed, if one wants the animals to sleep freely for long periods of time that allow pairing trials to be given, the animals must be placed in a familiar recording box that is not associated with fearful events such as electric shocks. In addition, if the animals are to be water deprived, as they were in our experimental conditions, a box in which water is usually available cannot be used. For all these reasons, the second-order conditioning procedure must be conducted outside of the apparatus used for acquisition and testing of the conditioned suppression response. Thus, the preliminary question that needs to be answered is whether or not a secondorder association can be accomplished in an external context distinctly different from that used for the first-order conditioning, and whether it can be expressed in a testing context different from that used for acquisition.

In Experiment 1, we developed an experimental paradigm in which the second-order conditioning procedure was carried out in one apparatus and the first-order conditioning and test phases took place in another. To minimize context changes, both apparatuses were located in the same experimental room. This experiment was under- 
taken only with waking animals, to determine whether the experimental conditions would allow the second-order conditioning to occur, as expressed by subsequent behavior.

\section{Method}

Animals. Naive male Wistar rats, weighing $250 \mathrm{~g}$ on arrival in the laboratory, served as subjects. They were housed in individual cages with wood shavings as bedding, in a temperature-controlled colony room under a 12:12-h light:dark cycle (8 a.m.-8 p.m. light). The rats were allowed unlimited access to food throughout the experiment. They received water freely until 1 week before the study was started.

Surgery. Each animal had a double earring permanently hooked to one ear; it was made with two looped silver wires $(0.4 \mathrm{~mm}$ in diameter, $2 \mathrm{~cm}$ in length) fixed to a light-weight plug ( $7 \times 5 \times 3 \mathrm{~mm})$. This bipolar stimulation electrode was used to apply mild ETS. The surgery was performed under general anesthesia (pentobarbital, $60 \mathrm{mg} / \mathrm{kg}$ i.p.) 2 weeks before the beginning of the experiment.

Apparatus. A first box (the "licking box") was used for the lick training, first-order conditioning, and testing phases. This box, $23 \times 23 \times 40 \mathrm{~cm}$, had three walls of opaque Perspex. A transparent ceiling and front wall allowed observation of the animal. A grid floor, mounted $5 \mathrm{~cm}$ above the bottom of the box, was composed of stainless steel rods $0.5 \mathrm{~cm}$ in diameter, spaced $1.5 \mathrm{~cm}$ center to center. The grid could be electrified by a scrambled-current shock source. A circular hole, $1 \mathrm{~cm}$ in diameter and $6 \mathrm{~cm}$ above the grid floor in the center of the left wall, gave access to a metal lick tube attached to a bottle of tap water placed outside the box. The orifice of the drinking spout was centered in the hole, $0.5 \mathrm{~cm}$ behind the wall. Licking was recorded through a contact relay circuit; contacts with the drinking spout (licks) were recorded when a circuit was completed with the grounded grid floor. The top of the box was equipped with a loudspeaker ( $5 \mathrm{~cm}$ in diameter). The stimulating cable connected to the ear of the animal was hung with rubber bands from a hook at the top of the box. The cable ran out the box through a centrally located aperture in the ceiling. This arrangement permitted undisturbed spontaneous behavior.

A second box (the "treatment box") was used for the secondorder conditioning phase. This opaque Armodur box, $25 \times 25 \times 60 \mathrm{~cm}$, was identical to the home cage except at the top. It had a transparent front door and translucent window in both sidewalls. The floor was layered with wood shavings. Food was freely available in the box. The top was equipped with a loudspeaker $(5 \mathrm{~cm}$ in diameter). Cables connected to the animal were relayed at the top of the box through a multichannel rotating connector.

The licking box and the treatment box were each enclosed in sound-attenuating material. Both were located in the same room (the experimental room), which was $4.5 \mathrm{~m}$ long and $2.5 \mathrm{~m}$ wide. The two boxes were distant from each other: one was placed along the left wall, near the back of the room, the other along the right wall, near the front. Ambient white noise was continuously present in the experimental room.

Electrotactile stimulation. During Phase 1 of the experiment, the intensity of earshock used during the subsequent experimental phases was determined for each animal. The behavioral responses to electric shocks to the ear were observed in each rat in its home cage, when it was awake and immobile. The ETS consisted of a square wave of $250-\mathrm{msec}$ duration. The intensities were varied and delivered in a random order. As in our previous experiments (Hars \& Hennevin, 1990; Hars et al., 1985), the chosen intensity induced a slight but reproducible head movement.

Procedure. A schedule of water deprivation was established over the 7 days before the start of the experiment proper. The water bottles were removed from the rats' home cages at 7 p.m. on the 1 st day, and on each subsequent day, they were presented for a fixed period of time between 6:30 and 7:30 p.m. This time period was $1 \mathrm{~h}$ on the 1 st day, $30 \mathrm{~min}$ on the $2 \mathrm{nd}, 15 \mathrm{~min}$ on the $3 \mathrm{rd}, 10 \mathrm{~min}$ on the 4th and 5 th days, and $5 \mathrm{~min}$ on the final day. Throughout the course of the experiment, the subjects continued to receive free access to water for 5-10 min (depending on individual body weight) each day, between 7 and 7:30 p.m. Under these conditions, animals were reduced to $80 \%$ of ad-lib body weight and were maintained at that level throughout the experiment.

The experiment proper began on the following day, Day 1. Table 1 summarizes the experimental design.

Phase 1. For 6 consecutive days, between 10 and 12 a.m., animals were trained to lick for water. On Day 1, they were placed individually for $5 \mathrm{~min}$ in the licking box with the drinking tube protruding $1 \mathrm{~cm}$ into the box. At the end of the 5 -min period, the animals were removed and returned to their home cages. This procedure was repeated on the next 5 days, except that the drinking spout was in its normal pulled outside position. From Day 3, a cable was connected to the ear of the rat when it was in the licking box. Total licks at the water spout were recorded on the second session and on each subsequent one. All the animals quickly learned to drink almost continuously during the 5-min period.

In the afternoon, between $1: 30$ and 6:30 p.m., the animals were progressively familiarized with the treatment box. They were placed individually in the box for a fixed period of time each day. This period was lengthened over days, from $30 \mathrm{~min}$ to $1 \mathrm{~h} 30 \mathrm{~min}$. On Day 4 and until the end of the experiment, a stimulation cable was connected to the ear of the animal when it was in the treatment box.

Phase 2. On Days 7 and 8, between 10 and 12 a.m., a classical conditioning procedure was carried out while the rats were engaged in licking. The CS (14.5 sec in duration) consisted of a train of

Table 1

Summary of the Paradigm Used in the Three Experiments

\begin{tabular}{|c|c|c|c|c|}
\hline Experiment & $\begin{array}{l}\text { Days } 1-6 \\
\text { Licking Box } \\
\text { Lick Training }\end{array}$ & $\begin{array}{c}\text { Days 7-8 } \\
\text { Licking Box } \\
\text { First-Order Conditioning }\end{array}$ & $\begin{array}{c}\text { Day } 9 \\
\text { Treatment Box } \\
\text { Second-Order Conditioning } \\
\end{array}$ & $\begin{array}{c}\text { Days } 10-12 \\
\text { Licking Box } \\
\text { Test } \\
\end{array}$ \\
\hline 1 & Licking & ETS-footshock pairing & $\begin{array}{l}\text { Group P: } 12 \text { tone-ETS pairings during waking } \\
\text { Group NP: } 12 \text { tones, } 12 \text { ETSs unpaired during waking } \\
\text { Group NS: no stimuli }\end{array}$ & Tone alone \\
\hline 2 & Licking & ETS-footshock pairing & $\begin{array}{l}\text { Group P-W: } 12 \text { tone-ETS pairings during waking } \\
\text { Group P-PS: } 30 \text { tone-ETS pairings during PS } \\
\text { Group P-SWS: } 30 \text { tone-ETS pairings during SWS } \\
\text { Group T: } 12 \text { tones alone during waking } \\
\text { Group NS: no stimuli }\end{array}$ & Tone alone \\
\hline 3 & Licking & ETS-footshock pairing & $\begin{array}{l}\text { Group P: } 3 \text { tone-ETS pairings during waking } \\
\text { Group ES: } 3 \text { ETSs alone during waking } \\
\text { Group NS: no stimuli }\end{array}$ & Tone alone \\
\hline
\end{tabular}


20 electrical stimulations to the ear: ETSs were $0.25 \mathrm{sec}$ in duration each; they were interspaced by 0.5 -sec intervals. The UCS was an electrical footshock ( $0.5 \mathrm{sec}$ in duration) delivered at CS offset. The ETS intensity was that previously assessed for each individual; the mean intensity was $130 \mu \mathrm{A}$ (range, 80-160 $\mu \mathrm{A}$ ). The intensity of footshock was adjusted for each animal; mean intensity was $0.30 \mathrm{~mA}$ (range, $0.26-0.34 \mathrm{~mA}$ ). At each session, the $300 \mathrm{th}$ lick initiated the first conditioning trial. There were four trials in Session 1 and three in Session 2, with a mean intertrial interval of $3 \mathrm{~min}$ (range, 2-5 $\mathrm{min}$ ). In each session, the animal was allowed 300 licks after the last trial and was then removed from the box and returned to its home cage. The rats progressively showed lick suppression to ETS presentation. Those that failed to exhibit a quasitotal suppression of licking during the ETS period (that is, those that made more than 6 licks during ETS presentation) in the two final trials of conditioning on Day 8 were discarded.

In the afternoon, each rat was adapted to the treatment box for a 2-h period.

Phase 3. The treatment phase of the experiment occurred on Day 9. First, subjects were allowed to recover their baseline lick rates so that meaningful suppression measures could be obtained in later sessions. In the morning, the animals were placed in the licking box for a 10-min period and were then returned to their home cages. Those that failed to emit at least 1,000 licks during the last $5 \mathrm{~min}$ of the session were eliminated. Twenty-two animals went on to the next stage of the experiment, making three groups balanced for both magnitude of lick suppression response in the last two trials of conditioning on Day 8 and spontaneous licking rate during the recovery session.

In the afternoon, the rats were placed for $2 \mathrm{~h}$ in the treatment box, where they were given different treatments according to the group to which they were assigned. For the animals in the experimental group (Group P, $n=8$ ), a $1000-\mathrm{Hz}$ tone $(80 \mathrm{~dB}$ of intensity, $6 \mathrm{sec}$ in duration) was paired with a train of eight ETSs (each $0.25 \mathrm{sec}$ in duration; 0.5-sec interval between ETSs). The first ETS was delivered $4 \mathrm{sec}$ after tone onset. For each individual, ETS intensity maintained the value used during the first-order conditioning. Each animal received, when it was awake and immobile, 12 tone-ETS pairings distributed into three series of four pairing trials each. The first series began at least 30 min after the placement of the rat in the box. Within a series, pairings were given at intervals ranging from 15 to $120 \mathrm{sec}$. Intervals between the series ranged from 10 to $30 \mathrm{~min}$. The animal was removed from the box at least 20 min after the third series. Two control groups were studied. Animals in Group NP $(n=7)$ were treated as those in Group P, except that tone and ETS presentations were never paired. Both characteristics and number of tones and ETSs, as well as their distribution over the 2-h period, were the same as for Group P. The animals in Group NS ( $n=7)$ received no stimulus presentations in the treatment box.

Phase 4. Lick suppression tests were conducted on Days 10-11 between 10 and 12 a.m. Nonreinforced tone presentations were superimposed on the licking instrumental baseline. In each session, $5 \mathrm{~min}$ long, the rats received two 15 -sec presentations of the 1000 $\mathrm{Hz} 80-\mathrm{dB}$ tone, initiated at $1 \mathrm{~min} 30 \mathrm{sec}$ and $4 \mathrm{~min}$ into the session.

Treatment of data. Stimulus presentations were controlled by a microcomputer, which also recorded the behavioral data. From Day 2 to Day 12, total licks were recorded for each session. On each trial of the test sessions, licks were counted during the $15 \mathrm{sec}$ of tone and during the $15 \mathrm{sec}$ preceding the tone. All statistical comparisons were carried out with analyses of variance (ANOVAs) performed on individual raw data (licks per $15 \mathrm{sec}$ ). For each test session, the mean number of licks during both tone presentations was compared with mean number of licks during both pretone periods in that session.

\section{Results and Discussion}

The baseline rates of licking were stable and equivalent among the three groups, which did not differ in mean number of licks during the $15-\mathrm{sec}$ pretone period in Test 1 $[F(2,19)<1]$ and in Test $2[F(2,19)=2.32$, n.s. $]$.

Each group exhibited lick decrement to tone presentation in both the first and the second testing sessions $(p<$ .001 for both sessions for Group $\mathrm{P} ; p<.025$ and $p<$ .05 for Group NP; $p<.05$ for both sessions for Group NS). But, as Figure 1 shows, the degree of lick rate suppression differed among the groups. A two-way ANOVA, with treatment group and period (pretone vs. tone) as the factors, revealed a significant interaction between these two factors in Test $1[F(2,19)=28.21, p<$ $.001]$ and in Test 2 as well $[F(2,19)=21.43, p<.001]$. Subsequent analyses showed that animals exposed to paired tone-ETS presentations exhibited greater lick suppression than did those in the two other groups. On both test days, tone-elicited suppression was more pronounced in Group $P$ than in Group NP [Test $1, F(1,13)=38.80$; Test 2, $F(1,13)=18.69 ; p<.001$ in both cases] or Group NS $[F(1,13)=51.79, F(1,13)=37.76$, respectively, $p<$ $.001]$. Lick suppression did not differ between group NP and Group NS $[F(1,12)<1$ and $F(1,12)=1.94$, respectively, n.s.].

Thus, all three groups displayed a suppression of licking in response to tone presentation. In control groups for which the tone either did not occur at all (Group NS) or had been explicitly unpaired with ETS (Group NP), the slight suppression likely reflects the unconditioned effects of the tone (i.e., the tone's ability to elicit an orienting response) and further, a generalized fear reaction to any salient stimulus presented in a context in which footshocks had been delivered previously. The lack of difference between the two control groups shows that the
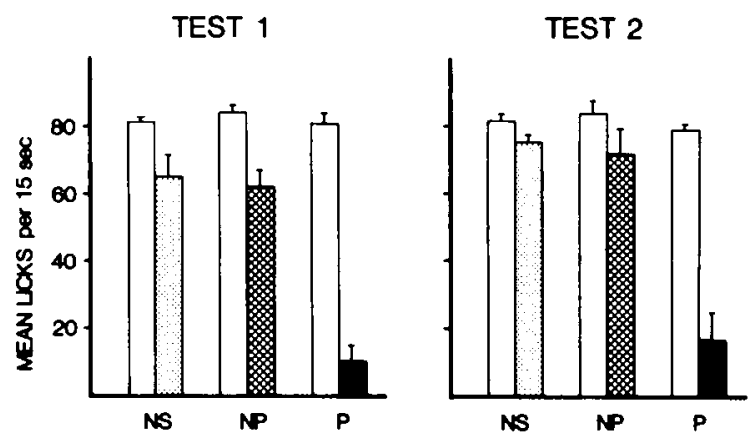

Figure 1. Mean number of licks $(+S E M)$, in each test session, during the 15-sec pretone period (white columns) and during the $15 \mathrm{sec}$ of tone. During the treatment phase of the experiment, Group NS experienced no stimulus presentations; Group NP was exposed to unpaired presentations of the tone and ETS; and Group P received 12 paired trials of the tone and ETS. Note the strong toneelicited suppression of licking in Group P. On the other hand, note that reactivity to the tone in Group NP did not differ from that in Group NS. 
pseudoconditioning procedure did not modify the normal suppressive effect of the tone. On the other hand, lick suppression response was strongly enhanced in animals that had received paired tone-ETS presentations. This greater suppression response to the tone was not due to some nonassociative effect, since it was not present after unpaired stimulus presentations. It can be regarded as a conditioned fear response to the tone, indicating that the animals had actually learned the association between the tone and ETS.

Therefore, these results allow a clear answer to the question addressed in the present experiment: the secondorder conditioning occurred and induced a profound suppression of licking at tone presentation, although it took place in an external context other than that used for the first-order conditioning and testing phases. In the next experiment, we questioned whether pairing the tone and ETS during sleep would likewise modify licking behavior in the presence of the tone at testing.

\section{EXPERIMENT 2}

The use of a pairing procedure during sleep involves several constraints. First, the intensity of the stimuli must be low enough so that they will be nonawakening. On the other hand, the intensity must be sufficient for the stimuli to be detected by the sleeping organism. We observed in pilot studies that behavioral reactivity to a stimulus presented during sleep varied over time within one phase of sleep. It tends to be higher at the beginning of the phase and to become lower as the phase goes on. Such a trend can be regarded as being the result of either a deeper sleeping state or a habituation process, or both. In agreement with our observations, it has been shown that when a tone of weak intensity is repeatedly presented during one PS phase, the auditory nerve potential shows a progressive decrement in amplitude as the episode of PS progresses (Velutti, Pedemonte, \& Garcia-Austt, 1989). Therefore, in previous experiments in which we introduced a cue stimulus during sleep (Hars \& Hennevin, 1987, 1990; Hars et al., 1985), we progressively increased the intensity of the stimulus. We used the same procedure in the present experiment. Within a given sleep phase, the intensity of both stimuli was very low on the first pairing trial, and it progressively increased as the sleep phase continued. This was repeated for each sleep phase studied.

Another constraint is that one has no control over the sequences of behavioral states which are quite variable in the rat. The durations of sleep phases and the intervals between sleep phases are unpredictable, especially in the case of paradoxical sleep. In some cases, a single PS phase was too short for more than one or two pairing trials, and, as a consequence, the stimulus intensity was very weak. To compensate, we increased the number of pairings given during sleep relative to that used in the preceding experiment.

To summarize, the second-order conditioning procedure that we employed during sleep differed in several ways from that used in Experiment 1: the intensity of both stimuli varied and was lower on the average; pairing trials were more numerous and were delivered over a longer period of time.

There were five treatment groups in the present study. Three experimental groups received tone-ETS pairings during paradoxical sleep, slow-wave sleep, or waking. The latter of these groups was included to ensure that the second-order conditioning could occur despite variable and weak intensity of stimuli. Two control groups were included. One received no stimulus presentations during the treatment phase of the experiment. The other experienced only tone presentations during the waking state, so that the test trials might give an accurate measure of the conditioned suppression generated by tone-ETS pairing in the experimental groups.

\section{Method}

Animals. The animals had the same characteristics as did those in Experiment 1, and they were treated in the same way. The only difference was that they were all implanted with three cortical and two neck-muscle electrodes so that we could detect their vigilance states. Silver extradural cortical electrodes (ECOG) were placed along the interhemispheric suture with a large frontoparietal contralateral derivation. Two silver electrodes were sewn through the dorsal neck muscles for the recording of electromyographic activity (EMG). As before, a double earring was permanently hooked to one ear of each rat.

Apparatus and recording. The apparatus was that used in Experiment 1. A Grass polygraph served for ECoG and EMG recording. During the treatment phase of the experiment, ECoG and EMG were permanently monitored so that the vigilance states could be recognized as they occurred. A trial was initiated only when the animal was in a well-defined state. The determination of states of sleep was based on classically used criteria: low-frequency, highamplitude ECOG activity and moderate tonic EMG activity for SWS; fast, low-voltage ECoG activity and no tonic EMG activity for PS.

Electrotactile stimulation. The intensity of earshock delivered during the subsequent experimental phases was determined for each animal in the same way as in Experiment 1. Two intensities were chosen: the lowest one ("low ETS") induced only a clear movement of the auricle; the highest ("high ETS") elicited a slight but reproducible head movement as in Experiment 1. During the secondorder conditioning phase, ETS intensity was progressively increased from a low ETS to a high ETS value. The mean difference between the lowest and the highest values was $50 \mu \mathrm{A}$.

Procedure. The animals were maintained on a water deprivation schedule in the same way as in Experiment 1. The general procedure was the same as before, and the experiment consisted of the same phases. The paradigm is summarized in Table 1.

After 6 days of preliminary lick training, waking animals underwent first-order conditioning in two sessions. Both the CS and the UCS had the same characteristics as in Experiment 1. The ETS intensity was the high ETS value previously assessed for each individual; the mean intensity was $125 \mu \mathrm{A}$ (range, 80-150 $\mu \mathrm{A}$ ). The mean intensity of footshock was $0.30 \mathrm{~mA}$ (range, $0.28-0.34 \mathrm{~mA}$ ). The animals that failed to exhibit a marked ETS-elicited suppression were eliminated.

On the morning of Day 9, the animals received one 10-min session for the recovery of licking. Those that failed to lick consistently were discarded. Thirty-nine animals went on to the next stage of the experiment, making five groups equalized both for the magnitude of lick suppression response in the two final trials of condi- 
tioning and for the lick rate during the recovery session. In the afternoon, each subject was placed in the treatment box for $2 \mathrm{~h}$ at least and was connected to recording and stimulating cables. ECoG and EMG activities were permanently monitored.

The experimental animals received tone-ETS pairings. As in Experiment 1 , a $1000-\mathrm{Hz}$ tone, $6 \mathrm{sec}$ long, was paired with a train of eight ETSs beginning $4 \mathrm{sec}$ after tone onset. Pairing trials were distributed among several series. The intensity of both the tone and the ETS increased progressively within each series. For the ETS, the lowest intensity used was the low ETS value previously assessed for each individual. Intensity increased progressively to reach, as far as possible, the level used during the first-order conditioning phase (high ETS). In each experimental group, the mean ETS intensity was $23 \%-25 \%$ lower than that used during the first-order conditioning. For the tone, the intensity was increased from 50 to $75 \mathrm{~dB}$; the mean intensity was $66 \mathrm{~dB}$. The animals in Group P-PS $(n=8)$ received 30 tone-ETS pairings during PS phases. After the onset of a PS phase, $15 \mathrm{sec}$ were allowed to elapse before the 1 st pairing trial. Within a phase, the intertrial interval varied from 20 to $30 \mathrm{sec}$. Trials were distributed among several PS phases, from 4 to 8 according to their duration. The intertrial interval could be up to $1 \mathrm{~h}$. The 30 pairing trials were delivered over a mean period of $2 \mathrm{~h} 30 \mathrm{~min}$ (range, $2 \mathrm{~h}$ to $3 \mathrm{~h} 30 \mathrm{~min}$ ). The animals in Group P-SWS ( $n=8$ ) received 30 tone-ETS pairings during phases of slow-wave sleep. Each animal of this group was yoked with an animal of Group P-PS with regard to intertrial intervals. The animals in Group P-W $(n=8)$ received 12 tone-ETS pairings during periods of wakefulness. Pairing trials were distributed into three series of 4 pairings each, over a period of $1 \mathrm{~h}$. Thus, these subjects were treated as those of Group P in Experiment 1, except that the ETS and tone intensities varied in the same way as they did during sleep.

The animals in Control Group T $(n=7)$ received 12 presentations of the tone alone while they were awake; these were delivered in three series of 4 tone presentations each, over a 1-h period. As in the preceding groups, the tone intensity was varied, with a mean of $65 \mathrm{~dB}$. The animals in Control Group NS $(n=8)$ were merely placed in the treatment box, where they remained for $2 \mathrm{~h}$. No stimuli were presented.

On the next 3 days, all subjects were tested for suppression to the tone. The tone intensity was $70 \mathrm{~dB}$. There were two test trials per session, delivered as in Experiment 1.

Any procedural details not specified above were the same as those in Experiment 1.

\section{Results and Discussion}

The baseline rates of licking were stable and did not differ among the five groups $[F(4,34)<1$ on each test day].

In terms of responses to tone presentation in Test 1 , Control Group $\mathrm{T}$, which had received presentations of tone alone on Day 9, was found to contrast with the other 4. Indeed, a within-group comparison showed no toneelicited suppression in these animals $[F(1,6)<1]$. By contrast, a significant suppression response to the tone occurred in each group that had received tone-ETS pairing $[p<.005$ or less in Groups $\mathrm{P}-\mathrm{W}, \mathrm{P}-\mathrm{PS}$, and P-SWS], as well as in Control Group NS, which had experienced no stimuli $[F(1,7)=6.44, p<.05]$. However, the degree of lick suppression rate differed among these four groups, as is indicated by a significant interaction between treatment group and period factors $[F(3,28)=$ $4.85, p<.01]$. From Figure 2, it is apparent that Group NS suppressed least, that Group P-W suppressed most, and that the two other groups had an intermediate level of suppression. A statistical analysis of the treatment group $\times$ period interaction supported this. Unlike the three other groups, Group NS did not significantly differ from Group $\mathrm{T}[F(1,14)=3.01$, n.s. $]$. Group P-W showed more suppression than did Group NS $[F(1,14)=$ $8.59, p<.01]$ or Group P-SWS $[F(1,14)=5.38, p<$ .05]. The difference between Groups P-W and P-PS did not reach the .05 significance level $[F(1,14)=3.30, p=$ .087]. Group P-PS showed somewhat more suppression than did Group NS $[F(1,14)=4.10, p=.059]$. In Group P-SWS, the degree of suppression differed neither from that in Group P-PS $[F(1,14)<1]$ nor from that in Group NS $[\mathrm{F}(1,14)=1.51$, n.s.].

Reactivity to the tone varied among the five groups in Test 2 as well, as indicated by a significant group $\times$ period interaction $[F(4,34)=6.98, p<.001]$. There was no suppression in either control group $[F(1,7)=1.99$, n.s., for Group NS; $F(1,6)=2.49$, n.s., for Group T]
TEST 1

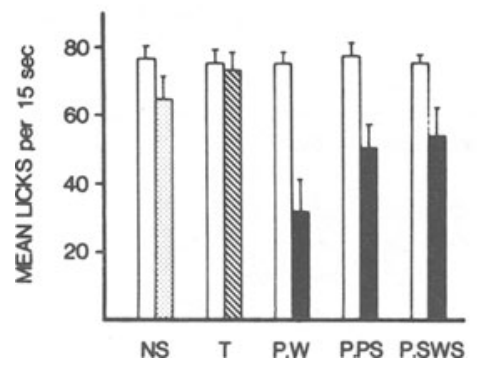

TEST 2

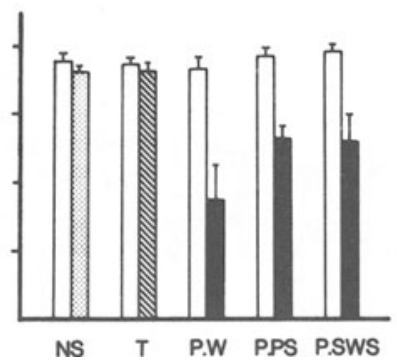

TEST 3

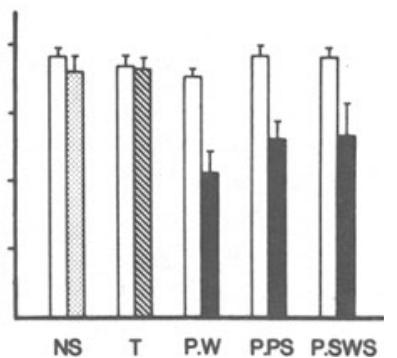

Figure 2. Mean number of licks $(+S E M)$, in each test session, during the 15-sec pretone period (white columns) and during the 15 sec of tone. During the treatment phase of the experiment, Group NS experienced no stimulus presentations; Group T was given presentations of tone alone; Group P received paired trials of the tone and ETS during waking (Group P-W), or paradoxical sleep (Group P-PS), or slow-wave sleep (Group P-SWS). Note that, in contrast with the two other groups, all three groups that received tone-ETS pairing, whether in wakefuiness or in sleep, exhibited a marked tone-elicited suppression of licking that was maintained over all three test sessions. 
as opposed to the three experimental groups $(p<.005$ for Groups P-W and P-PS; $p<.025$ for Group P-SWS). But this time, the degree of suppression rate was not significantly different among these three groups $[F(2,21)=$ 1.03, n.s.].

The same pattern of results was observed for Test 3 : no suppression in the control groups $[F(1,7)=1.49$ for Group NS, and $F(1,6)<1$ for Group T]; suppression in the three experimental groups $(p<.001$ for Group $\mathrm{P}-\mathrm{W}$, $p<.005$ for Group P-PS, and $p<.01$ for Group PSWS); and no difference among these three groups $[F(2,21)<1]$.

Thus, reactivity to the tone at testing differed between control and experimental subjects. The two groups that had not received tone-ETS pairings showed either no suppression or little suppression of licking, depending on whether they had been previously exposed to the tone or not. Comparison of these two groups indicates the magnitude of unconditioned responses to the tone. The animals without prior tone experience (Group NS) showed some lick decrement to tone presentation at testing as did those in Experiment 1, indicating that presentation of a novel stimulus by itself can elicit a moderate suppression of licking. But this unconditioned suppression response became habituated more rapidly in Experiment 2 than in Experiment 1 . This might be viewed as a consequence of the fact that tone intensity at testing was lower in Experiment 2 than it was in Experiment 1. The animals that had been exposed to prior tone presentations (Group $\mathrm{T}$ ) showed no suppression at testing, which suggests that prior repeated exposures to the tone had brought about the habituation of unconditioned responses.

On the other hand, all three groups that had received paired tone-ETS presentations exhibited a marked suppression that was maintained over all three test sessions. The results from Group P-W replicate those from Group $P$ in Experiment 1. Again, the pairing of the tone with ETS for waking subjects resulted in a strong conditioned fear in the presence of the tone at testing, indicating that the effects of second-order conditioning transferred from one context to another. Nonetheless, the animals in the present experiment showed somewhat less suppression than that shown by the animals in Experiment 1. Two procedural changes might account for this difference: at pairing, the mean intensity of both the tone and the ETS was lower than that used in Experiment 1; at testing, the tone intensity was lower than in Experiment 1 . Thus, the smaller level of suppression observed in the present experiment might reflect reduction either in the associative value of the tone or in unconditioned response to the tone, or in both.

The main result of Experiment 2 is that the groups that received tone-ETS pairing during sleep also exhibited a substantial lick suppression when exposed to the tone at testing. In both groups, this response was strong enough to be maintained over all 3 test days. This is most likely the consequence of paired presentations of the tone and ETS during sleep. However, despite all of the precautions that we had taken, a few ECoG arousals were polygraphically detected in the course of pairing. Most of them were transient, but some were more enduring. They occurred sometimes at the beginning of a pairing trial, during tone presentation, and sometimes after tone offset, during ETS delivery. The mean number of arousals was 0.75 (range, 0-2) for Group P-PS and 1 (range, 0-2) for Group P-SWS. Although some of them occurred even when both stimuli were of low intensity, there is no way to ascertain whether the arousals were elicited by stimulus presentation or were spontaneous awakenings. The question of course arises as to whether tone-ETS association was learned during the trials given while the animals were actually awake. The next experiment was aimed at assessing such a possibility.

\section{EXPERIMENT 3}

The purpose of this study was to examine to what extent the second-order conditioning can be established when very few pairing trials were given to waking subjects.

In the preceding experiment, in the worst cases, two awakenings were observed in the course of pairing; and sometimes awakening occurred when the stimulus intensity was very low. The conditions in Experiment 3 were still more propitious for learning, since the experimental animals received three tone-ETS pairings with highintensity stimuli. Given that in Experiment 2 some awakenings had occurred after tone offset, another group of subjects was exposed to three presentations of ETS alone. A third group receiving no stimulus presentations served as a control group.

\section{Method}

Subjects and Apparatus. The subjects had the same characteristics as in Experiment 1. They were maintained on a water deprivation schedule as in Experiments 1 and 2. The apparatus and stimuli were also the same as before.

Procedure. The general procedure was similar to that previously used, and the experiment consisted of the same phases. Table 1 summarizes the paradigm.

After 6 days of preliminary lick training, all rats received firstorder conditioning in two sessions. Animals that failed to exhibit a marked suppression to ETS presentation at conditioning, as well as animals that failed to lick reliably at the subsequent lick recovery session, were discarded. Twenty-seven animals went on to the next stage of the experiment, making 3 groups, each with 9 subjects, matched for ETS-elicited suppression at conditioning and for lick rates at the recovery session.

On the afternoon of Day 9 , each subject was placed in the treatment box for $2 \mathrm{~h}$ and connected to the ear-stimulating cable. The animals in Group P received three tone-ETS pairings. The intensity was fixed for both stimuli, $80 \mathrm{~dB}$ for the tone, and that used during first-order conditioning for the ETS (the mean intensity was $130 \mu \mathrm{A})$. The animals in Group ES received only ETS to the ear. They were given three presentations of ETS, each consisting of an eight-ETS train. The mean ETS intensity and distribution of ETS presentations were as those for Group P. The animals in Group NS received no stimulus presentations. On the next 3 days, all subjects were tested for suppression to the 80-dB tone.

Any procedural details not specified above were the same as they were for Experiment 1. 


\section{Results and Discussion}

It is apparent from Figure 3 that test performances were similar among the three groups. Statistical analysis confirms this. There was no difference among groups in baseline rates of licking $[F(2,24)<1$ for each session]. Each group showed some lick decrement to tone presentation $[p<.05$ or less for each group in each session]. The result of interest is that the degree of suppression was quite equivalent among the groups: no significant group $\times$ period interaction was found $[F(2,24)<1$ for each test day].

Thus, there was no sign of any difference among the three groups. The lick decrement to tone presentation in each group reflects the normal unconditioned effects of the tone. Neither three tone-ETS pairings nor three exposures to the ETS alone affected the strength of the suppression. Therefore, these results provide no support for the idea that in Groups P-PS and P-SWS of Experiment 2 acquisition of the tone-ETS association could have occurred during the one or two trials given when the animals woke up.

\section{GENERAL DISCUSSION}

The main findings from the present experiments can be summarized as follows. The pairing of a tone with ETS previously used as the CS in aversive training induced a marked fear response to the tone as assessed by suppression of licking behavior. This response was observed whether the second-order pairing was carried out during waking or during sleep.

The strong lick suppression exhibited by subjects that had received tone-ETS pairings while awake can be regarded as a result of associative learning. The fact that these subjects differed strongly from those exposed to unpaired tone-ETS presentations clearly indicates that the suppression of drinking was not due to some nonassociative effect but was a consequence of second-order conditioning. The effect of conditioning was strong enough to transfer across contexts, since the second-order associa- tion was established in a box other than that used for firstorder conditioning and was behaviorally expressed later in a box other than that which was used for acquisition. It is arguable that the two boxes actually constituted discriminably different environments for the rat. The fact that they were both located in the same room might have increased the effective similarity of the two contexts and thus the probability of generalization between them. Nevertheless, the two boxes differed from one another in a number of ways. More particularly, in one box there was a grid floor and water was available, whereas in the other box, the floor was covered with wood shavings and water was not available. It is more than likely that such cues were sufficiently relevant to allow the animal to regard the two boxes as different contexts. It remains possible that the strength of the second-order responding was reduced by the change of context, but we have no data on this point. Yet our results do give some support to the proposition that behavioral response to a second-order Pavlovian CS remains well preserved even if the external context is changed.

In addition, the effect of second-order pairing does not appear to be confined to the internal context in which it took place. After tone-ETS pairing in a state of vigilance (i.e., during sleep), the tone elicited a lick suppression response when it was later presented in another state (i.e., during waking). This efficacy of pairing cannot be at tributed to what was experienced during the very few trials on which arousals were detected. The results of Experiment 3 clearly demonstrated that even three paired trials of tone and ETS given to animals fully awake were not sufficient to induce any change in reactivity to the tone at testing. Thus, the tone-elicited suppression exhibited by Groups P-PS and P-SWS in Experiment 2 was indeed a consequence of stimulus presentation during sleep. According to the conventional concept of state dependency (Overton, 1964), information may be retrievable only under the same physiological state as that of acquisition. The changes in state that occur between sleeping and waking may be expected to produce a state-dependent retrieval
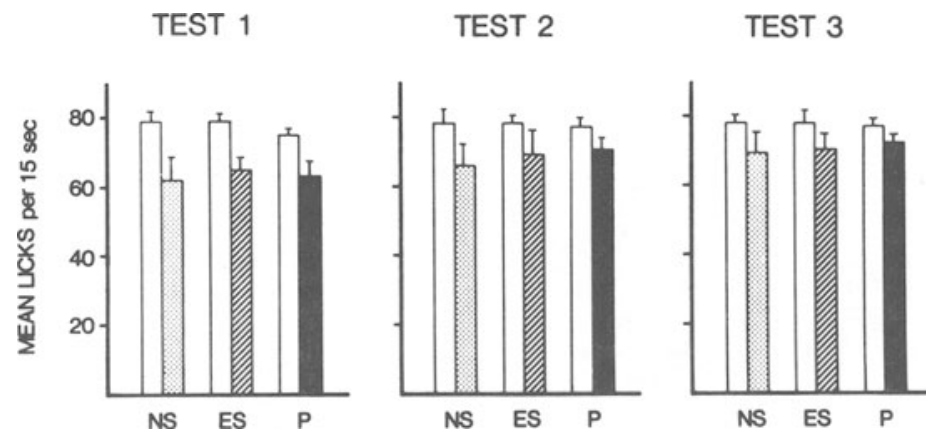

Figure 3. Mean number of licks ( $+S E M)$, in each test session, during the 15sec pretone period and during the 15 sec of tone. During the treatment phase of the experiment, Group NS experienced no stimulus presentations; Group ES was given presentations of ETS alone; Group P received three paired trials of the tone and ETS. Note that reactivity to the tone at testing was quite equivalent among the three groups. 
failure. This type of hypothesis has already been proposed to account for the well-known difficulty of recalling dreams (Goodenough, 1978). Such a deficit cannot be excluded in the present study: it could contribute to the lower suppressing power of the tone after pairing during sleep relative to that resulting from pairing during the waking state. But, if it did exist, this state-dependent impairment was small. The change in vigilance state did not prevent what was experienced during sleep from being expressed in waking behavior. This conclusion is consonant with previous results, which show that a cuing treatment experienced during PS or SWS is effective in modifying the performance of waking animals (Hars \& Hennevin, 1987; Hars et al., 1985). Furthermore, a hippocampal conditioned response established during PS was transferred to the waking state (Maho \& Bloch, in press). Moreover, there is evidence that learning can be expressed when the internal state of the organism is quite different from that during acquisition. It has been shown, for example, that animals can learn a specific CS-UCS association under ketamine anesthesia, and further, that this acquisition can be expressed behaviorally several days later when animals are tested for retention in a conditioned suppression paradigm (Edeline \& Neuenschwander-El Massioui, 1988). Similar results have been obtained when training was conducted under deep barbiturate anesthesia, in rats receiving epinephrine administration (Gold, Weinberger, \& Sternberg, 1985; Weinberger, Gold, \& Sternberg, 1984). Finally, it has been shown that the effect of second-order pairing is well preserved despite change in the internal state. For instance, motivational changes resulting from modifications in conditions of satiation have no impact on second-order responding, but such changes affect firstorder performance (Holland \& Rescorla, 1975).

We found no difference in test performance between animals that had received the paired tone and ETS during PS or SWS. The effectiveness of pairing during PS agrees with a number of studies that support the idea that PS is a state that provides the conditions for information processing to take place. Elsewhere, we have developed arguments in favor of this proposal (Bloch, Hennevin, \& Leconte, 1979; Hennevin \& Hars, 1985). Indeed, wakefulness and PS are fundamentally equivalent functional states (for a recent discussion, see Llinas \& Paré, 1991). We have provided evidence which suggests that the processes that occur during postlearning PS might be of the same kind as those acting just after the acquisition of information (Bloch, Hennevin, \& Leconte, 1977; Hennevin, Hars, \& Bloch, 1989). Some data also suggest that the processing of new information is performed during PS by mechanisms that are fundamentally identical to those that occur during waking (Maho \& Bloch, in press). On the other hand, the effectiveness of pairing during SWS is somewhat more surprising. Indeed, SWS is a brain state quite different from the PS and waking states, and some controversy exists regarding the possibilities of information processing during this state of sleep. It is clear that SWS does permit some processing to take place, since the organism in SWS is quite able to discriminate between stimuli according to their relevance. Detection of significant stimuli is generally associated with arousal from sleep, but there have been examples of discriminative responses made without awakening (Ciancia et al., 1980; Sano et al., 1982; Yehuda et al., 1979). However, some studies have shown that a difference in information processing might exist between SWS and PS. For example, presentation of a cue stimulus during PS improves subsequent retention performance (Hars et al., 1985), whereas presentation of the same stimulus during SWS impairs performance (Hars \& Hennevin, 1987). Maho and Bloch (in press) have reported that a hippocampal conditioned cellular response developed as a result of pairing two intracerebral stimulations during PS, whereas it did not after pairing in SWS. But there are some problems in the interpretation of the latter result. The authors emphasized that they mostly recorded hippocampal theta cells. Such cells have been shown to change their firing rate with behavioral state: they fire much faster during waking and PS, and they present low activity during SWS (Delacour, 1980; Ranck, 1973; Suzuki \& Smith, 1985). Thus, one possibility is that the lack of effectiveness of the pairing procedure conducted in SWS resulted from an incapacity of the theta cells to increase their firing rate during SWS and not from an inability of the brain in SWS to process information. Therefore, to what extent the processing of information might be different between SWS and PS remains to be determined. In the present experiments, we found no evidence of any difference; the suppression of drinking in response to the tone presented at testing was equivalent, whether pairing had been conducted during SWS or PS.

The magnitude of tone-elicited suppression was smaller after pairing in sleep, relative to that induced by pairing during wakefulness, especially in the first test session. Several possibilities might account for this smaller response.

First, we have already mentioned the possibility of a state-dependent retrieval failure. The effect of the pairing procedure would be similar in sleep and in wakefulness, but it might not be expressed as easily when a change in state occurs at the time of testing.

A second possibility might be that too many pairing trials were given during sleep. A greater number of trials were indeed given during sleep than during wakefulness, so that a sufficient number of trials in which intensity of stimuli was high enough could be presented in sleep. But it has been reported that in a second-order pairing procedure, in contrast with a first-order one, responding to the second-order stimulus showed a peak level after a few trials and then fell off as pairing continued (Holland, 1977; Rescorla, 1980). Given this, one might speculate that some form of overtraining inhibition process would have begun to develop in sleep as a result of the increased number of pairing trials.

Third, one obvious possibility to account for the smaller suppressive effect of the tone observed in subjects that had experienced pairing while asleep is that the mechanisms that govern the processing of information would be less powerful during sleep than they are in the waking 
state. In addition, the effectiveness of pairing in sleep was possibly reduced because of the lowered stimulus value of the tone and the ETS. It is trivial to say that incoming sensory inputs from the outside world are attenuated during sleep. Since the tone and ETS were delivered at the same intensity during waking and sleep, it is likely that their stimulus value was lowered when they were presented in sleep. It is well documented that the effectiveness of a pairing procedure normally improves as the intensity of either the CS or the UCS increases (Mackintosh, 1974). Hence, the pairing of stimuli might have been more effective with waking than with sleeping subjects.

A final possibility to consider is that the process that occurs during sleep might be different from that which occurs during wakefulness. The main question is whether or not the lick suppression to the tone exhibited by subjects exposed to tone-ETS pairing during sleep can be regarded as a result of associative learning that has taken place during sleep. One source of support for this interpretation is the fact that, in Experiment 1, unpaired presentations of the tone and ETS to waking subjects were shown to yield very little suppression to the tone at testing. The performance of those animals never differed from that of control rats that had experienced no stimulus presentations at all. On the other hand, the animals in Experiment 2 that had received paired presentations of the tone and ETS while asleep showed a more profound and more enduring suppression of drinking than did the control rats exposed to no stimuli. Nevertheless, because of the absence of control groups with unpaired presentations of tone and ETS during sleep, one cannot totally rule out the possibility that some strengthening operation, albeit not the tone-ETS contingency, could have developed during sleep. Yet that such a process could have occurred in sleep, while it did not in wakefulness, and further, that it could have been strong enough in sleep to express itself in the waking state over all three test sessions, would be somewhat perplexing. However this may be, it is clear that the experience of the tone and the ETS during sleep was capable of modifying subsequent waking behavior in the presence of the tone. It is generally agreed that learning has occurred when a particular experience at one time produces an outcome at a later time which would be absent without that experience. From this viewpoint, the present study suggests that some form of learning did occur during sleep.

In conclusion, after a tone had been paired during sleep with slight ETS to the ear (previously used as the CS in aversive training), that tone induced marked suppression of licking when it was subsequently presented to the waking animals. This effect was reliable, since it was expressed over all three test sessions. Thus, the present study does suggest that what was experienced during sleep could transfer to the waking state and find behavioral expression. Such results should encourage further investigation of information-processing abilities during sleep, and the second-order conditioning procedure provides an interesting and efficient tool to explore this topic.

\section{REFERENCES}

BALSAM, P. D. (1985). The functions of context in learning and performance. In P. D. Balsam \& A. Tomie (Eds.), Context and learning (pp. 1-21). Hillsdale, NJ: Erlbaum

BLoCh, V., HeNnevin, E., Leconte, P. (1977). Interaction between post-trial reticular stimulation and subsequent paradoxical sleep in memory consolidation processes. In R. R. Drucker-Colin \& J. L. McGaugh (Eds.), Neurobiology of sleep and memory (pp. 255-272). New York: Academic Press.

Bloch, V., Hennevin, E., \& Leconte, P. (1979). Relationship between paradoxical sleep and memory processes. In M. A. Brazier (Ed.), Brain mechanisms in memory and learning: From the single neuron to man (pp. 329-343). New York: Raven Press.

Bouton, M. E., Bolles, R. C. (1985). Context, event-memories and extinction. In Context and learning (pp. 133-166). Hillsdale, NJ: Erlbaum

Buendia, N., Sierra, G., Goode, M., a Segundo, J. P. (1963). Conditioned and discriminatory responses in wakeful and in sleeping cats. Electroencephalography \& Clinical Neurophysiology, 24(Suppl.), 199-218.

Ciancia, F., Trigona-Leisinger, M. C., Bloch, V. (1980). Etude, chez le chat, de la réactivité aux stimulus auditifs signifiants en fonction des niveaux de vigilance. Archives laliennes de Biologie, 118 375-385.

Delacour, J. (1980). Conditioned modifications of arousal and unit activity in the rat hippocampus. Experimental Brain Research, 38, 95-101.

Delacour, J. (1982). Associative and non-associative changes in unit activity of the rat hippocampus. Brain Research Bulletin, 8, 367-373.

Dillon, R. F., W WebB, W. B. (1965). Threshold of arousal from "activated" sleep in the rat. Joumal of Comparative \& Physiological Psychology, 59, 446-447.

Edeline, J. M., Neuenschwander-El Massioui, N. (1988). Retention of CS-US association learned under ketamine anesthesia. Brain Research, 457, 274-280.

ESTES, W. K., SkINNER, B. F. (1941). Some quantitative properties of anxiety. Journal of Experimental Psychology, 29, 390-400.

Gold, P. E., Weingerger, N. M., \& Sternberg, D. B. (1985), Epinephrine-induced learning under anesthesia: Retention performance at several training-testing intervals. Behavioral Neuroscience, 99, 1019-1022

Goodenough, D. R. (1978). Dream recall: History and current status of the field. In A. M. Arkin, J. S. Antrobus, \& S. J. Ellman (Eds.), The mind in sleep: Psychology and psychophysiology (pp. 113-140). Hillsdale, NJ: Erlbaum.

Hall, G., Channell, S. (1985). Differential effects of contextual change on latent inhibition and on the habituation of an orienting response. Joumal of Experimental Psychology: Animal Behavior Processes, 11, 470-481.

Halperin, J. M., lorio, L. C. (1981). Responsivity of rats to neutral and danger-signaling stimuli during sleep. Behavioral \& Neural Biology, 33, 213-219.

Hars, B., Hennevin, E. (1987). Impairment of learning by cueing during postleaming slow-wave sleep in rats. Neuroscience Letters, 79, $290-294$.

Hars, B., Hennevin, E. (1990). Reactivation of an old memory during sleep and wakefulness. Animal Leaming \& Behavior, 18, 365-376.

Hars, B., Hennevin, E., \&asques, P. (1985). Improvement of leaming by cueing during postlearning paradoxical sleep. Behavioural Brain Research, 18, 241-250.

Hennevin, E., Hars, B. (1985). Post-learning paradoxical sleep: A critical period when new memory is reactivated? In B. E. Will, P. Schmitt, \& J. C. Dalrymple-Alford (Eds.), Brain plasticity, learning and memory (pp. 193-203). New York: Plenum.

Hennevin, E., HARS, B., \& BLoCH, V. (1989). Improvement of learning by mesencephalic reticular stimulation during postleaming paradoxical sleep. Behavioral \& Neural Biology, 51, 291-306.

Holland, P. C. (1977). Conditioned stimulus as a determinant of the form of the Pavlovian conditioned response. Journal of Experimental Psychology: Animal Behavior Processes, 3, 77-104. 
Holland, P. C., \& Rescorla, R. A. (1975). The effects of two ways of devaluing the unconditioned stimulus after first- and second-order appetitive conditioning. Journal of Experimental Psychology: Animal Behavior Processes, 1, 355-363.

Izquierdo, I., WyrWicka, W., Sierra, G., \& Segundo, J. P. (1965). Établissement d'un réflexe de trace pendant le sommeil naturel chez le chat. In A. M. Mounier (Ed.), Actualités neurophysiologiques (pp. 277-296). Paris: Masson.

JOHNEN, M. (1987). The sleeping rat shows signs of orienting response to neutral stimuli. Psychophysiology, 24, 104-111.

LELORD, G., \& MAHO, C. (1969). Modifications des activités corticales et thalamiques au cours d'un conditionnement sensoriel: I. Localisation des réponses et variations avec la vigilance. Electroencephalography \& Clinical Neurophysiology, 27, 258-269.

Llinas, R. R., \& PARE, D. (1991). Of dreaming and wakefulness. Neuroscience, 44, 521-535.

Lovibond, P. F., Preston, G. C., Mackintosh, N. J. (1984). Context specificity of conditioning, extinction and latent inhibition. Journal of Experimental Psychology: Animal Behavior Processes, 10, 360-375.

MaCkInTOSH, N. J. (1974). The psychology of animal learning. London: Academic Press.

М^но, C., Bцосн, V. (in press). Responses of hippocampal cells can be conditioned during paradoxical sleep. Brain Research.

Maho, C., Hennevin, E., Hars, B., \& Polncheval, S. (1991). Evocation in paradoxical sleep of a hippocampal conditioned cellular response acquired during waking. Psychobiology, 19, 193-205.

ONIANI, T. N., \& LoRTKIPANIDZE, N. D. (1985). Retrieval and extinction of conditioned reflexes during sleep. In T. N. Oniani (Ed.), Neurophysiology of motivation, memory and sleep-wakefulness cycle (pp. 214-233). Tbilisi, Georgia, U.S.S.R.: Metsniereba.

OverToN, D. A. (1964). State-dependent or "dissociated"' learning produced with pentobarbital. Journal of Comparative Physiological Psychology, 57, 3-12.

Overton, D. A. (1985). Contextual stimulus effects of drugs and internal states. In P. D. Balsam \& A. Tomie (Eds.), Context and learning (pp. 357-384). Hillsdale, NJ: Erlbaum.

Pavlov, I. P. (1927). Conditioned reflexes. London: Oxford University Press.

RANCK, J. B. (1973). Studies on single neurons in dorsal hippocampal formation and septum in unrestrained rats: I. Behavioral correlates and firing repertoires. Experimental Neurology, 41, 462-531.

Rescorla, R. A. (1980). Pavlovian second-order conditioning. Hillsdale, NJ: Erlbaum.
Rescorla, R. A., Durlach, P. J., \& Grau, J. W. (1985). Contextual learning in Pavlovian conditioning. In P. D. Balsam \& A. Tomie (Eds.), Context and learning (pp. 23-56). Hillsdale, NJ: Erlbaum.

RowlaND, V. (1957). Differential electroencephalographic response to conditioned auditory stimuli in arousal from sleep. Electroencephalography \& Clinical Neurophysiology, 9, 585-594.

RUDOLPH, K. (1987). Habituation of the rat's respiratory response to auditory stimulation during sleeping and waking. Psychobiology, 15, 272-276.

Sano, A., Matsumoto, J., \& Ishikawa, N. (1982). Conditional reflex during sleep in cats. Tokushima Journal of Experimental Medicine, 29, $11-20$.

SASAKI, H., \& YoshII, N. (1984). Conditioned responses in the visual cortex of dogs: II. During sleep. Electroencephalography \& Clinical Neurophysiology, 58, 448-456.

Segundo, J. P., Roig, J. A., \& Sommer-Smith, J. A. (1959). Conditioning of reticular formation stimulation effects. Electroencephalography \& Clinical Neurophysiology, 11, 471-484.

SiEgel, J., \& LANGLEY, T. D. (1965). Arousal threshold in the cat as a function of sleep phase and stimulus significance. Experientia, 21, $740-741$.

Suzukı, S. S., \&MITH, G. K. (1985). Single-cell activity and synchronous bursting in the rat hippocampus during waking behavior and sleep. Experimental Neurology, 89, 71-89.

Trigona, M. C., Ciancia, F., BLoCh, V. (1968). La profondeur du sommeil paradoxal chez le chat: Seuil de stimulation réticulaire et détection des stimuli sonores signifiants ou non. Journal de Physiologie, 60, 559-560.

Van Twyver, H., \& Garrett, W. (1972). Arousal threshold in the rat determined by "meaningful" stimuli. Behavioral Biology, 7, 205-215.

Velutti, R., Pedemonte, M., \& Garcia-Austt, E. (1989). Correlative changes of auditory nerve and microphonic potentials throughout sleep. Hearing Research, 39, 203-208.

Weinberger, N. M., Gold, P. E., SternberG, D. B. (1984). Epinephrine enables Pavlovian fear conditioning under anesthesia. Science, 223, 605-607.

Yehuda, S., Chorover, S. L., \& Carasso, R. L. (1979). Habituation and transfer during sleep in cats. Intermational Joumal of Neuroscience, 9, 225-227.

(Manuscript received November 19, 1991; revision accepted for publication February 26, 1992.) 